\title{
Urinary expression of genes involved in DNA methylation and histone modification for diagnosis of bladder cancer in patients with asymptomatic microscopic haematuria
}

\author{
ILARIA LUCCA ${ }^{1,2}$, SEBASTIAN L. HOFBAUER ${ }^{1}$, ANDREA HAITEL $^{3}$, MARTIN SUSANI ${ }^{3}$, \\ SHAHROKH F. SHARIAT ${ }^{1,4-7}$, TOBIAS KLATTE ${ }^{1}$ and MICHELA DE MARTINO ${ }^{1}$
}

\author{
${ }^{1}$ Department of Urology, Medical University of Vienna, A-1090 Vienna, Austria; ${ }^{2}$ Department of Urology, \\ Centre Hospitalier Universitaire Vaudois, 1011 Lausanne, Switzerland; ${ }^{3}$ Department of Pathology, \\ Medical University of Vienna; ${ }^{4}$ Department of Urology, Comprehensive Cancer Center, Medical University of Vienna, \\ Vienna General Hospital; ${ }^{5}$ Karl Landsteiner Society, Urology and Andrology, A-1090 Vienna, Austria; \\ ${ }^{6}$ Department of Urology, Weill Cornell Medical College, New York, NY 10011; ${ }^{7}$ Department of Urology, \\ University of Texas Southwestern Medical Center, Dallas, TX 75390, USA
}

Received October 11, 2018; Accepted April 25, 2019

DOI: $10.3892 / 01.2019 .10330$

\begin{abstract}
The aim of the present study was to identify and test a urine marker panel of genes involved in DNA methylation and histone modification for the detection of urothelial carcinoma of the bladder (UCB). RNA samples obtained from the voided urine of 227 patients with asymptomatic microscopic haematuria (AMH) were analysed. Gene array analysis was performed on 18 randomly selected cDNA samples, which revealed that histone deacetylase 9 (HDAC9), HDAC3, $t R N A$ (cytosine-5-)-methyltransferase1 and DNA methyltransferase 1 were differentially expressed between patients with UCB and control subjects. Subsequently, reverse transcription-quantitative polymerase chain reaction analysis was employed to test the performance of the identified four-gene panel on the remaining 209 cDNA samples. In this targeted discovery cohort, all four genes were significantly associated with UCB on univariable analyses [each odds ratio $(\mathrm{OR})>2$, $\mathrm{P}<0.05$ ], but only HDAC3 was significant following multivariable analysis $(\mathrm{OR}=2.8, \mathrm{P}=0.011)$. The addition of $H D A C 3$ to a base risk factor model improved its accuracy by $1.4 \%$. These data suggest that urinary $H D A C 3$ is associated with the presence of UCB in patients with $\mathrm{AMH}$; however, HDAC3 improved the accuracy of the established risk factors only to a marginal extent.
\end{abstract}

Correspondence to: Dr Michela de Martino, Department of Urology, Medical University of Vienna, Währinger Gürtel 18-20, A-1090 Vienna, Austria

E-mail: demartino@gmx.net

Key words: urothelial, methylation, histone modification, DNA, RNA, histone deacetylase 3

\section{Introduction}

Urothelial carcinoma of the bladder (UCB) is the second most common urological cancer with 430,000 new cases diagnosed worldwide annually (1). A total of $80 \%$ of the patients present with non-muscle invasive bladder cancer (NMIBC) (2), which has a recurrence rate of $\sim 50 \%$ (3). NMIBC requires intensive, invasive follow-up and repeat interventions, making it one of the most costly malignancies per patient (4).

Cystoscopy is the established standard tool for the workup of patients with asymptomatic microscopic haematuria (AMH) (5), but it is invasive, costly and imperfect. $\mathrm{AMH}$ is a major challenge for health care providers due to its high prevalence, limited healthcare resources and low adherence to unpractical algorithms in current guidelines (6). Ultimately, $<5 \%$ of patients investigated are subsequently diagnosed with UCB (7). Intense research has therefore been undertaken to identify easier, better, faster, more cost-effective urinary markers for UCB detection, aiming to single out the patients who may truly benefit from cystoscopy, and avoiding unnecessary testing and possible iatrogenic complications (8). Indeed, several urine-based markers have been shown to have a potential use as a non-invasive adjunct to cystoscopy for the detection of UCB (9-15), but none has yet fulfilled the criteria to be recommended for routine care (6).

Epigenetic changes play a key role in UCB pathogenesis and progression. Indeed, UCB is defined by multiple epigenetic events that inactivate known tumour suppressors through DNA methylation and histone modification (16). In human DNA, methylation is a molecular process targeting $\mathrm{CpG}$ dinucleotides. Most genes have unmethylated $\mathrm{CpGs}$ in their 5' regulatory region. DNA methyltransferases (DNMTs) catalyse the methylation of these $\mathrm{CpGs}$, thereby inducing transcriptional silencing, leading to suppressed tumour growth and cancer cell invasion (17).

Given the unmet need for urinary biomarkers in the diagnostic setting and the pivotal role of epigenetic modifications in 
UCB carcinogenesis, we hypothesised that urinary epigenetic biomarkers are associated with the presence of UCB. To test this hypothesis, we identified and validated urinary markers involved in DNA methylation and histone modification for UCB detection in patients with AMH.

\section{Materials and methods}

Study cohort. We prospectively collected voided urine samples from 300 patients with AMH between 2013 and 2016. All individuals were evaluated by urinalysis, cystoscopy, ultrasound or computed tomography scan of the urinary tract and bladder biopsies, when appropriate. None had suspected urinary tract infection, known IgA nephropathy, indwelling catheters, visible haematuria, known UCB, concurrent or previous upper tract urothelial carcinoma, renal cell carcinoma or prostate cancer. The patients provided written informed consent and all the procedures were carried out under the Good Scientific Practice Standards. The Ethics Committee of the Medical University of Vienna approved the present study (Study ID 1162/2013).

Pathological evaluation. Two expert genitourinary pathologists processed and reviewed all the pathological specimens according to standard protocols. Pathological stage was assigned according to the 2009 TNM classification (18), and grade according to the $2004 \mathrm{WHO} /$ ISUP classification (19).

Urine processing and RNA quality control. All analysed urine samples were negative for leukocytes and nitrite, but positive for erythrocytes. Midstream-voided urine samples $(50 \mathrm{ml})$ were collected in a sterile cup prior to instrumentation or contrast studies. For each sample, a unique identification number was assigned prior to laboratory processing, and the laboratory staff were blinded to the suspected diagnosis. Total RNA was purified using the ZR Urine RNA Isolation Kit (Zymo Research Corporation) and RNA quantity and quality were assessed with an Agilent 2100 Bioanalyser (Agilent Technologies, Inc.) according to the manufacturer's instructions. Of the 300 RNA samples, 73 were excluded from further studies due to low quantity and/or quality. Total RNA $(0.5 \mu \mathrm{g})$ from the 227 remaining samples was used to synthesize complementary DNA (cDNA) using Taqman Reverse Transcription Reagents (Applied Biosystems; Thermo Fisher Scientific, Inc.).

Gene discovery by qPCR array. The commercially available TaqMan ${ }^{\circledR}$ Array Human DNA Methylation and Transcriptional Repression (cat. no. 4414127; Thermo Fisher Scientific, Inc.) was used, which is a pre-made array and includes 24 target genes associated with DNA methylation and transcriptional repression, and 8 reference genes the expression of which we wanted to evaluate. The 32 genes and their associated data are listed in Table SI. The experiment aimed to select differentially expressed target genes and a set of stably expressed reference genes for normalization in the validation study. A total of 18 RNA samples were selected in a random manner using the 'sampling' library in R 3.5, including 7 obtained from the urine of individuals without cancer and 11 from UCB patients. The two populations were matched for sex and smoking rates, but the control group was significantly younger (median (range), 55 (44-75) vs. 71 (48-84), P=0.03) compared with the UCB group. Each gene was analysed in triplicate. A total of $5 \mu 1$ TaqMan ${ }^{\circledR}$ Fast Advanced Master Mix (Thermo Fisher Scientific, Inc.) and $0.5 \mu \mathrm{l}$ cDNA were brought to a total volume of $10 \mu \mathrm{l}$ by adding DNAse and RNAse-free water, and the final mixture was added to the TaqMan ${ }^{\circledR}$ Array plates. The plates were briefly centrifuged at $112 \mathrm{RCF}$ and subsequently run with the following conditions: $50^{\circ} \mathrm{C}$ for $2 \mathrm{~min}, 95^{\circ} \mathrm{C}$ for $20 \mathrm{sec}$ and 40 cycles at $95^{\circ} \mathrm{C}$ for $3 \mathrm{sec}$ and at $60^{\circ}$ for $30 \mathrm{sec}$. Gene expression was evaluated by reverse transcription-quantitative polymerase chain reaction (RT-qPCR) analysis on a QuantStudio $^{\text {TM }} 7$ Flex Real-Time PCR System (Applied Biosystems; Thermo Fisher Scientific, Inc.).

Target genes with a minimum 2-fold change between cases and controls and between patients with low- vs. high-grade UCB were selected for the validation study. Reference gene expression stability was analysed based on average pairwise variation as the M-value. Candidate reference genes were ranked by stepwise exclusion of genes with the highest $\mathrm{M}$-value. To determine the possible need for reference genes for normalisation, the pairwise variation was calculated and 0.2 was selected as a cut-off value, below which additional reference genes are not required (20).

Gene validation by $q P C R$. The four target genes [DNMT1, histone deacetylase 3 (HDAC)3, HDAC9, tRNA (cytosine-5-)-methyltransferase 1 (TRDMT1)] exhibiting differential expression were subsequently analysed by standard RT-qPCR on cDNA synthesised from RNA of our independent cohort constituted by the 209 remaining urine samples. Among the eight candidate endogenous reference genes, $A C T B$, HPRTI and GUSB exhibited the highest expression stability and were therefore used in subsequent validation experiments. The TaqMan ${ }^{\circledR}$ Gene Expression Assays (Applied Biosystems; Thermo Fisher Scientific, Inc.) were used for DNMT1 (assay ID Hs00154749_m1), HDAC3 (Hs00187320_m1), HDAC9 (Hs00206843_m1), TRDMT1 (Hs00189402_m1), GUSB (Hs99999908_m1), HPRT1 (Hs99999909_m1) and ACTB (Hs99999903_m1). The RT-qPCR mixture was prepared for each sample as follows: $10 \mu 1$ TaqMan ${ }^{\mathrm{TM}}$ Universal PCR Master Mix, no AmpErase ${ }^{\mathrm{TM}}$ UNG (Applied Biosystems; Thermo Fisher Scientific, Inc.), $1 \mu 1$ TaqMan Gene Expression Assay and $3 \mu \mathrm{l}$ cDNA were brought to a total volume of $20 \mu \mathrm{l}$ by adding DNase and RNase-free water. Each sample was assayed in triplicate. The RT-qPCR reactions were analysed on the system used for arrays analysis with the following conditions: $90^{\circ} \mathrm{C}$ for $10 \mathrm{~min}$ and 40 cycles at $95^{\circ} \mathrm{C}$ for $15 \mathrm{sec}$ followed by $60^{\circ} \mathrm{C}$ for $1 \mathrm{~min}$. Amplification plots were assessed using the detection software SDS v2.0.1 (Applied Biosystems; Thermo Fisher Scientific, Inc.) to confirm that the quantification cycle $\left(\mathrm{C}_{\mathrm{q}}\right)$ value corresponded with the midpoint of the logarithmic amplification. Data were then processed using DataAssist v3.0.1 (Applied Biosystems; Thermo Fisher Scientific, Inc.). $\mathrm{C}_{\mathrm{q}}$ values not detected in the measurable range $\left(\mathrm{C}_{\mathrm{q}}>40\right)$ were considered as not determinable.

Statistical analysis. Categorical variables are presented as numbers and percentages, and continuous variables as median and interquartile range (IQR). Group differences in categorical variables and continuous variables were analyzed 
with chi-square tests and Mann-Whitney U tests, respectively. The proportions of patients with detectable HDAC3, HDAC9, TRDMT1 and DNMT1 were compared using Chi-squared tests. For quantitative analysis, the relative expression of HDAC3, HDAC9, TRDMT1 and DNMT1 was determined by normalising against their levels for the three reference genes $\left(\Delta \mathrm{C}_{\mathrm{q}}\right.$ method). Inter-class fold changes were calculated using the $2^{-\Delta \Delta \mathrm{Cq}}$ method (21). Odds ratios (OR) and $95 \%$ confidence intervals $(95 \% \mathrm{CI})$ were obtained from univariable and multivariable logistic regression models. The full multivariable model combined all significant variables of the univariable analyses, and the reduced model was obtained after including variables that had a P-value of $<0.1$. The area under the curve (AUC) quantified the predictive accuracy. Statistical testing was two-sided and a P-value $<0.05$ was considered statistically significant. Analyses were all conducted with R 3.5 (The R Project for Statistical Computing).

\section{Results}

Discovery experiments. The gene array included 24 target genes associated with DNA methylation and transcriptional repression, as well as 8 reference genes. It was run on 18 randomly selected patients (11 UCB cases and 7 controls). HDAC9 and TRDMT1 were found to be differentially expressed between cases and controls (fold change 3.5 and 2.6, respectively) (Table SI)

Among UCB cases, 7 had low-grade and 4 had high-grade disease. DNMT1 and $H D A C 3$ exhibited a higher expression rate in high-grade vs. low-grade UCB (fold change 2.1 and 2.2, respectively). Tumour stage, smoking status and sex did not exhibit relevant differences between cases and controls or between high- and low-grade tumours.

Targeted discovery experiments/validation. The expression of HDAC3, HDAC9, TRDMT1 and DNMT1 was analysed in cDNA samples derived from the 209 remaining urine samples. The characteristics of the 209 patients in this targeted discovery cohort are shown in Table I.

The controls were younger $(\mathrm{P}<0.001)$, were more likely to be female $(\mathrm{P}=0.02)$ and less likely to be smokers compared with the UCB group $(\mathrm{P}=0.008)$. In the UCB group $(\mathrm{n}=117)$, 113 patients (97\%) were diagnosed with NMIBC and $4(3 \%)$ with muscle-invasive UCB. A total of 78 cases had low-grade UCB (67\%) and 39 had high-grade UCB (33\%).

HDAC3, HDAC9, TRDMT1 and DNMT1 were detected $\left(\mathrm{C}_{q} \leq 40\right)$ in $97(83 \%), 64(56 \%), 66(55 \%)$ and $77(67 \%)$ of the UCB patients and in $60(65 \%), 34(36 \%), 30(33 \%)$ and $38(40 \%)$ of the controls, respectively $(\mathrm{P}=0.003, \mathrm{P}=0.011, \mathrm{P}=0.001$ and $\mathrm{P}<0.001$, respectively). The presence of $H D A C 3$ was associated with smoking status $(\mathrm{P}=0.007)$ and the presence of $D N M T 1$ exhibited a weak association with UCB grade $(\mathrm{P}=0.02)$. No differences were found regarding sex, age or carcinoma in situ, and there was no significant association between the expression quantity of $H D A C 3, H D A C 9, T R D M T 1$ or $D N M T 1$ and any of the variables or outcomes.

All four genes were significantly associated with the detection of UCB on univariable logistic regression models (each $\mathrm{P}<0.05$; Table II). On the full multivariable model, none of the four tested urine markers reached statistical significance.
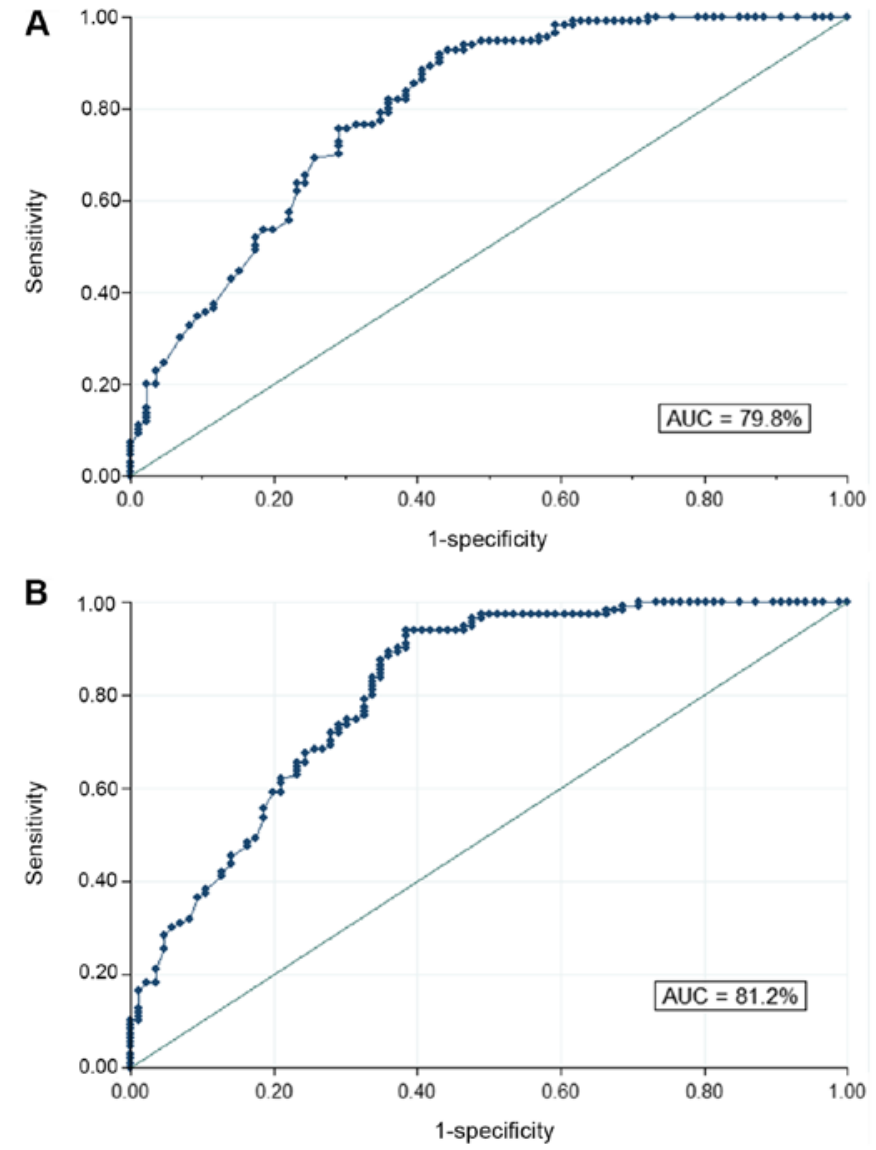

Figure 1. (A) Receiver operating characteristic curve based on routine risk factors for urothelial carcinoma of the bladder (age, sex, smoking status). (B) The addition of histone deacetylase to these routine risk factors improved the AUC by $1.4 \%$. AUC, area under the curve.

Using a backward selection multivariable analysis based on the full model, age $(\mathrm{P}<0.001)$, smoking status $(\mathrm{P}=0.042)$ and HDAC3 $(\mathrm{P}=0.011)$ remained independently associated with the presence of UCB. Addition of $H D A C 3$ to a base model for prediction of UCB with age, sex and smoking status improved the AUC by $1.4 \%$ (79.8 vs. $81.2 \%$, Fig. 1), with a sensitivity and specificity of 89 and $63 \%$, respectively, at the optimal cut-off. Combination analysis of various genes did not achieve a greater improvement in AUC (data not shown). The sensitivities, specificities, positive and negative predictive values of all four markers are reported in Table SII. Presence of HDAC3 was not associated with any other tumour characteristics (Table SIII).

\section{Discussion}

DNA methylation and histone modification genes play a key role in UCB development and progression (16), but only limited data are available on their expression in urine samples (22). Specifically, HDACs deacetylase the amino acid lysine on histone proteins, thereby rendering the chromatin structure inactive (23). Expression of HDACs is altered in multiple cancers (24). In UCB, the expression of HDACs was shown to be deregulated, and expression quantity has been associated with higher grade and a higher risk of progression (25). Overall, however, data on the urinary expression of DNMTs and $H D A C s$ as diagnostic markers in UCB are scarce. 
Table I. Characteristics of the 209 patients used as target discovery cohort.

\begin{tabular}{|c|c|c|c|c|}
\hline Variable & Total $\mathrm{n}$ & Cases & Controls & P-value \\
\hline $\mathrm{n}$ & 209 & 117 & 92 & - \\
\hline Sex, n (\%) & & & & 0.02 \\
\hline Female & $42(20)$ & $17(15)$ & $25(27)$ & \\
\hline Male & $167(80)$ & $100(85)$ & $67(73)$ & \\
\hline Age, median (IQR) & $67(53-75)$ & $72(64-78)$ & $56.5(34-69)$ & $<0.001$ \\
\hline Smoking status, n (\%) & & & & 0.008 \\
\hline Non-smokers & $73(36)$ & $32(28)$ & $41(48)$ & \\
\hline Current and former smokers & $123(59)$ & $78(63)$ & $45(37)$ & \\
\hline T stage, $\mathrm{n}(\%)$ & & & & - \\
\hline pTis & $4(3)$ & $4(3)$ & - & \\
\hline pTa & $81(69)$ & $81(69)$ & - & \\
\hline pT1 & $28(24)$ & $28(24)$ & - & \\
\hline pT2 & $4(3)$ & $4(3)$ & - & \\
\hline Grade, n (\%) & & & & - \\
\hline Low grade & $78(67)$ & $78(67)$ & - & \\
\hline High grade & $39(33)$ & $39(33)$ & - & \\
\hline Concomitant CIS, n (\%) & $15(13)$ & $15(13)$ & - & - \\
\hline Size, n (\%) & & & & - \\
\hline$<3 \mathrm{~cm}$ & $99(84)$ & $99(84)$ & - & \\
\hline$\geq 3 \mathrm{~cm}$ & $18(16)$ & $18(16)$ & - & \\
\hline Multifocality, n (\%) & $48(41)$ & $48(41)$ & - & - \\
\hline
\end{tabular}

P-values comparing the distribution between cases and controls are based on chi-square tests (sex, smoking status) and U tests (age). $\mathrm{IQR}$, interquartile range; CIS, carcinoma in situ.

Table II. Univariable and multivariable logistic regression models.

\begin{tabular}{|c|c|c|c|c|c|c|c|c|c|}
\hline \multirow[b]{2}{*}{ Variable } & \multicolumn{3}{|c|}{ Univariable model } & \multicolumn{3}{|c|}{ Multivariable full model } & \multicolumn{3}{|c|}{ Multivariable reduced model } \\
\hline & OR & $95 \% \mathrm{CI}$ & P-value & OR & $95 \% \mathrm{CI}$ & P-value & OR & $95 \%$ CI & P-value \\
\hline Age & 1.08 & $1.05-1.10$ & $<0.001$ & 1.08 & $1.05-1.10$ & $<0.001$ & 1.08 & $1.05-1.11$ & $<0.001$ \\
\hline Female sex & 0.45 & $0.23-0.90$ & 0.025 & 0.50 & $0.19-1.33$ & 0.17 & - & - & - \\
\hline Smoker & 2.22 & $1.23-4.00$ & 0.008 & 1.95 & $0.92-4.14$ & 0.08 & 2.14 & $1.03-4.45$ & 0.042 \\
\hline$H D A C 3$ presence & 2.59 & $1.36-4.93$ & 0.004 & 2.27 & $0.91-5.71$ & 0.08 & 2.80 & $1.26-6.19$ & 0.011 \\
\hline$H D A C 9$ presence & 2.06 & $1.18-3.60$ & 0.011 & 0.87 & $0.36-2.09$ & 0.75 & - & - & - \\
\hline$T R D M T 1$ presence & 2.67 & $1.51-4.72$ & 0.001 & 1.49 & $0.62-3.60$ & 0.37 & - & - & - \\
\hline$D N M T 1$ presence & 2.74 & $1.56-4.81$ & $<0.001$ & 1.33 & $0.57-3.11$ & 0.51 & - & - & - \\
\hline
\end{tabular}

HDAC, histone deacetylase; TRDMT, tRNA (cytosine-5-)-methyltransferase 1; DNMT1, DNA methyltransferase 1; OR, odds ratio; CI, confidence interval.

In the present study, we used pre-made gene arrays to analyse the expression of candidate genes associated with DNA methylation and transcriptional repression, and subsequently investigated those with differential expression in an independent set of patients. Among all genes, HDAC3 was found to be independently associated with UCB. Furthermore, it improved the accuracy of a multivariable base model, albeit only to a marginal extent of $1.4 \%$. However, there is no generally accepted cut-off in AUC increase that is considered clinically significant.

TRDMT1 and HDAC9 were found to be upregulated in UCB patients compared with controls. To the best of our knowledge, TRDMT1 (syn. DNMT2) has not yet been described as a potential marker for UCB. TRDMT1 upregulation was detected in several tumour samples, and it may be involved in gene silencing and RNA-mediated epigenetic 
heredity $(26,27)$. Similarly, altered expression of $H D A C 9$ has not been associated with the presence of UCB; however, its overexpression has been described in multiples cancers, and appears to promote tumour cell growth and cell cycle progression (28-30), all of which are considered key steps in UCB development and progression. In the present study, both genes were found to be overexpressed in UCB samples, although they failed to demonstrate an independent association on the multivariable analysis. This may be due to the small sample of patients analysed, limiting the statistical power.

DNMT1, conversely, has been previously shown to be overexpressed in UCB. In an immunohistochemical study on 130 UCB samples, DNMT1 immunoreactivity was higher in UCB samples compared with controls (31). This is in agreement with our observation that DNMT1 is more frequently detected in urine samples from UCB patients. Moreover, DNMT1 has been found to be associated with more advanced tumour stage (24\% expression in $\leq \mathrm{T} 1$ vs. 55\% in T2-T4 tumours) (31). While we did not observe an association of urinary DNMT1 with stage, it is important to note that only 4 patients had muscleinvasive UCB. However, differential expression according to tumour grade was observed. Finally, recent findings support a possible promising role of DNMT1 as early biomarker for UCB (32).

The genes differentially expressed in the first part of our study were further analysed in an independent group of 209 UCB patients. While all four genes were found to be significantly associated with the detection of UCB on univariable analyses, only $H D A C 3$ remained significantly associated on the multivariable analysis. $H D A C 3$ is part of the class I HDAC family, which also includes $H D A C 1, H D A C 2$ and $H D A C 8$. Deregulated expression of class I HDACs has been described in UCB, and HDAC inhibitors reduce UCB cell proliferation in vitro (33). Data on the role of HDACs in UCB are inconsistent. While the majority of the studies report overexpression of HDACs in cancers cells, some data suggest that elevated class I HDAC expression may improve prognosis in selected tumours (34). While Junqueira-Neto et al (35) observed higher $H D A C 3$ levels in low-grade tumours compared with controls, others found no differences when data were analysed at the protein level (36). In the present study, the quantity of urinary HDAC3 expression was lower in low-stage, low-grade, unifocal and small-sized UCB, although the differences were not statistically significant (data not shown). These data suggest a possible positive prognostic role of $H D A C 3$ overexpressed in UCB, which must be evaluated in further studies.

No significant association was found between overexpression of the four genes and any of the clinical variables. This implies that the urinary markers are able to detect UCB irrespective of stage and grade, which may be an important prerequisite for a routinely applied urine test.

There were certain limitations to the present study. Following RNA purification, $25 \%$ of RNA samples were excluded due to low quantity/poor quality. This may represent a problem in routine clinical practice, although constant improvements in RNA purification are achieved. Another limitation was patient selection. Indeed, although the cohort was well-selected, the overall number of patients was limited.
Moreover, the majority of patients included in this study were affected by UCB, making this patient sample not representative of a typical AMH population. Of the 4 genes, only $H D A C 3$ was associated with UCB on the multivariable reduced analysis. However, the other markers may improve their role in UCB detection if more patients are analysed.

Finally, adding HDAC3 to the base model achieved only a small improvement in UCB detection, minimising its potential impact on routine clinical practice. Further external validation studies with a larger population are required before $H D A C 3$ can be considered as a potential diagnostic marker in patients with AMH. The methylation status of specific substrates of each of these 4 genes may also be measured in order to support our data.

In conclusion, the data presented herein suggest that urinary $H D A C 3$ is associated with the presence of UCB in patients with $\mathrm{AMH}$ following adjustment for the effects of standard variables. However, HDAC 3 expression improves the accuracy of established risk factors only to a marginal extent.

\section{Acknowledgements}

Not applicable.

\section{Funding}

The present study was supported by the development fund of the CHUV-University Hospital, the European Urological Scholarship Program and the Anniversary Fund of the Oesterreichische Nationalbank (project no. 15362).

\section{Availability of data and materials}

The datasets used and analysed during the present study are available from the corresponding author on reasonable request.

\section{Authors' contributions}

IL, MDM and TK conceived and designed the study, analysed and interpreted the results, conducted the statistical analyses and drafted the manuscript. IL, SLH, AH, MS, SFS, MDM and TK acquired the data. TK, MDM, SLH, AH, MS and SFS critically revised the manuscript for important intellectual content. MDM and TK supervised the study.

\section{Ethics approval and consent to participate}

Patients provided written informed consent and all procedures were carried out under the Good Scientific Practice Standards. The Ethics Committee of the Medical University of Vienna approved the present study (Study ID 1162/2013).

\section{Patient consent for publication}

Patients provided informed consent.

\section{Competing interests}

The authors declare that they have no competing interests. 


\section{References}

1. Mahdavifar N, Ghoncheh M, Pakzad R, Momenimovahed Z and Salehiniya H: Epidemiology, incidence and mortality of bladder cancer and their relationship with the development index in the world. Asian Pac J Cancer Prev 17: 381-386, 2016.

2. Ederer IA, Lucca I, Hofbauer SL, Haidinger M, Haitel A, Susani M, Shariat SF and Klatte T: Histopathology and prognosis of de novo bladder tumors following solid organ transplantation. World J Urol 33: 2087-2093, 2015.

3. Leitner CV, Ederer IA, de Martino M, Hofbauer SL, Lucca I, Mbeutcha A, Mathieu R, Haitel A, Susani M, Shariat SF and Klatte T: Dynamic prognostication using conditional recurrence and progression estimates for patients with nonmuscle invasive bladder cancer. J Urol 196: 46-51, 2016.

4. Svatek RS, Hollenbeck BK, Holmäng S, Lee R, Kim SP, Stenzl A and Lotan Y: The economics of bladder cancer: Costs and considerations of caring for this disease. Eur Urol 66: 253-262, 2014.

5. Sountoulides P, Mykoniatis I and Metaxa L: Non-visible asymptomatic haematuria: A review of the guidelines from the urologist's perspective. Expert Rev Anticancer Ther 17: 203-216, 2017.

6. Schmitz-Dräger BJ, Kuckuck EC, Zuiverloon TC, Zwarthoff EC Saltzman A, Srivastava A, Hudson MA, Seiler R, Todenhöfer T, Vlahou A, et al: Microhematuria assessment an IBCN consensus-Based upon a critical review of current guidelines. Urol Oncol 34: 437-451, 2016.

7. Tan WS, Sarpong R, Khetrapal P, Rodney S, Mostafid H, Cresswell J, Hicks J, Rane A, Henderson A, Watson D, et al: Can renal and bladder ultrasound replace computerized tomography urogram in patients investigated for microscopic hematuria? J Urol 200: 973-980, 2018.

8. Bensalah K, Montorsi F and Shariat SF: Challenges of cancer biomarker profiling. Eur Urol 52: 1601-1609, 2007.

9. de Martino M, Lucca I, Mbeutcha A, Wiener HG, Haitel A, Susani M, Shariat SF and Klatte T: Carbonic anhydrase IX as a diagnostic urinary marker for urothelial bladder cancer. Eur Urol 68: 552-554, 2015.

10. de Martino M, Shariat SF, Hofbauer SL, Lucca I, Taus C, Wiener HG, Haitel A, Susani M and Klatte T: Aurora A Kinase as a diagnostic urinary marker for urothelial bladder cancer. World J Urol 33: 105-110, 2015.

11. Hofbauer SL, de Martino M, Lucca I, Haitel A, Susani M, Shariat SF and Klatte T: A urinary microRNA (miR) signature for diagnosis of bladder cancer. Urol Oncol 36: 531.e1-531.e8, 2018.

12. Chang Y, Xu J and Zhang Q: Microplate magnetic chemiluminescence immunoassay for detecting urinary survivin in bladder cancer. Oncol Lett 14: 4043-4052, 2017.

13. Lin T, Liu Z, Liu L, Yang L, Han P, Zhang P and Wei Q: Prospective evaluation of fluorescence in situ hybridization for diagnosing urothelial carcinoma. Oncol Lett 13: 3928-3934, 2017.

14. Xu X, Li P, Fu D, Wei Z, Xu S, Xu F, Tian F, Ge J, Zhang Z and Cheng W: Combined use of urinary Survivin detection and liquid-based cytology for the early diagnosis of bladder urothelial carcinoma. Oncol Lett 15: 7739-7743, 2018.

15. Yang Y, Xu J and Zhang Q: Detection of urinary survivin using a magnetic particles-based chemiluminescence immunoassay for the preliminary diagnosis of bladder cancer and renal cell carcinoma combined with LAPTM4B. Oncol Lett 15: 7923-7933, 2018.

16. Li HT, Duymich CE, Weisenberger DJ and Liang G: Genetic and epigenetic alterations in bladder cancer. Int Neurourol J 20 (Suppl 2): S84-S94, 2016

17. Lyko F: The DNA methyltransferase family: A versatile toolkit for epigenetic regulation. Nat Rev Genet 19: 81-92, 2018.

18. Sobin LH, Gospodarowicz MK and Wittekind C (eds): International Union Against Cancer: TNM Classification of Malignant Tumours, 7th Edition. Wiley-Blackwell, Chichester, 2009 .
19. Miyamoto H, Miller JS, Fajardo DA, Lee TK, Netto GJ and Epstein JI: Non-invasive papillary urothelial neoplasms: The 2004 WHO/ISUP classification system. Pathol Int 60: 1-8, 2010.

20. Vandesompele J, De Preter K, Pattyn F, Poppe B, Van Roy N, De Paepe A and Speleman F: Accurate normalization of real-time quantitative RT-PCR data by geometric averaging of multiple internal control genes. Genome Biol 3: RESEARCH0034, 2002.

21. Livak KJ and Schmittgen TD: Analysis of relative gene expression data using real-time quantitative PCR and the 2(-Delta Delta C(T)) method. Methods 25: 402-408, 2001.

22. Sapre N, Anderson PD, Costello AJ, Hovens CM and Corcoran NM: Gene-based urinary biomarkers for bladder cancer: An unfulfilled promise? Urol Oncol 32: 48.e9-17, 2014.

23. Bannister AJ and Kouzarides T: Regulation of chromatin by histone modifications. Cell Res 21: 381-395, 2011.

24. Hull EE, Montgomery MR and Leyva KJ: HDAC Inhibitors as epigenetic regulators of the immune system: Impacts on cancer therapy and inflammatory diseases. Biomed Res Int 2016: $8797206,2016$.

25. Poyet C, Jentsch B, Hermanns T, Schweckendiek D, Seifert HH, Schmidtpeter M, Sulser T, Moch H, Wild PJ and Kristiansen G: Expression of histone deacetylases 1,2 and 3 in urothelial bladder cancer. BMC Clin Pathol 14: 10, 2014.

26. Forbes SA, Beare D, Gunasekaran P, Leung K, Bindal N, Boutselakis H, Ding M, Bamford S, Cole C, Ward S, et al: COSMIC: Exploring the world's knowledge of somatic mutations in human cancer. Nucleic Acids Res 43: D805-D811, 2015.

27. Jeltsch A, Ehrenhofer-Murray A, Jurkowski TP, Lyko F, Reuter G, Ankri S, Nellen W, Schaefer M and Helm M: Mechanism and biological role of Dnmt 2 in nucleic acid methylation. RNA Biol 14: 1108-1123, 2017.

28. Gil VS, Bhagat G, Howell L, Zhang J, Kim CH, Stengel S, Vega F, Zelent A and Petrie K: Deregulated expression of HDAC9 in B cells promotes development of lymphoproliferative disease and lymphoma in mice. Dis Model Mech 9: 1483-1495, 2016.

29. Rastogi B, Raut SK, Panda NK, Rattan V, Radotra BD and Khullar M: Overexpression of HDAC9 promotes oral squamous cell carcinoma growth, regulates cell cycle progression, and inhibits apoptosis. Mol Cell Biochem 415: 183-196, 2016.

30. Lapierre M, Linares A, Dalvai M, Duraffourd C, Bonnet S, Boulahtouf A, Rodriguez C, Jalaguier S, Assou S, Orsetti B, et al: Histone deacetylase 9 regulates breast cancer cell proliferation and the response to histone deacetylase inhibitors. Oncotarget 7: 19693-19708, 2016.

31. Wu CT, Wu CF, Lu CH, Lin CC, Chen WC, Lin PY and Chen MF: Expression and function role of DNA methyltransferase 1 in human bladder cancer. Cancer 117: 5221-5233, 2011.

32. Zhang H, Qi D, Li J, Peng T, Yang L, Yuan J, Zhang Y, Hu Y, Su J, Que B, et al: A novel regulatory circuit of miR-152 and DNMT1 in human bladder cancer. Oncol Rep 40: 1803-1812, 2018.

33. Pinkerneil M, Hoffmann MJ, Kohlhof H, Schulz WA and Niegisch G: Evaluation of the therapeutic potential of the novel isotype specific HDAC inhibitor 4SC-202 in urothelial carcinoma cell lines. Target Oncol 11: 783-798, 2016.

34. Weichert W: HDAC expression and clinical prognosis in human malignancies. Cancer Lett 280: 168-176, 2009.

35. Junqueira-Neto S, Vieira FQ, Montezuma D, Costa NR, Antunes L, Baptista T, Oliveira AI, Graça I, Rodrigues Â, Magalhães JS, et al: Phenotypic impact of deregulated expression of class I histone deacetylases in urothelial cell carcinoma of the bladder. Mol Carcinog 54: 523-531, 2015.

36. Niegisch G, Knievel J, Koch A, Hader C, Fischer U, Albers P and Schulz WA: Changes in histone deacetylase (HDAC) expression patterns and activity of HDAC inhibitors in urothelial cancers. Urol Oncol 31: 1770-1779, 2013. 